\title{
Design and Implementation of Single-Layer Symmetric Micro-Transformers
}

\author{
Fahimullah Khan, Yong Zhu, Junwei Lu, and Jitendra Pal \\ Queensland Micro- and Nanotechnology Centre, Griffith University, Nathan, QLD 4111, Australia
}

In this paper, interleaved planar symmetric micro-transformers are implemented with single metal layer for the first time in the standard Metal-multi-user micro-electro-mechanical process (MUMP). Bonding wires are used for leading the inner terminal connections out to the outer terminal of the spiral square transformers. Two designs of micro-transformers with different dimensions are fabricated. The functionality of the devices was tested for very high frequency and ultra high frequency ranges using cascade ground signal ground probes and compared with the simulation results. The maximum efficiency of $80.65 \%$ has been achieved by $S 1$ micro-transformer design, and the maximum efficiency of $S 2$ micro-transformer is $76.9 \%$ at 1 GHz. The total footprint of $S 1$ and $S 2$ designs is 1.32 and $0.37 \mathrm{~mm}^{2}$, respectively.

Index Terms-Bonding wires, COMSOL multi-physics, on-wafer calibration, symmetric micro-transformers.

\section{INTRODUCTION}

W ITH the advancement of miniaturized portable products, the demand for on-chip efficient power supplies is growing day-by-day. Transformer is still the most effective solution for signal isolation and power transfer. Therefore, a lot of research is still being carried out on miniaturizing high power density and efficient transformers with special focus on isolated dc-dc converters for on-chip power supplies.

Several studies have been reported on different kinds of micro-transformer with various structures and fabrication techniques. The solenoidal micro-transformer has been presented in [1]-[3]. The problems with these transformers are the complex fabrication processes with their two-step core deposition to achieve uniaxial anisotropy. The spiral-type conductor micro-transformer is easy to fabricate with either circular [4], square [5], or race-track shape [6]. The race-track pot core transformer is renowned for high-power handling and magnetic anisotropy. In [6], the core is made of alloy with low resistivity, which induces eddy-current losses in the core. Furthermore, hysteresis losses and saturation flux density are complex problems in magnetic core transformers. These losses are frequency dependent, and increase with frequencies, resulting in a decrease in overall efficiency. The magnetic core could also compromise the signal isolation rating of the transformer [7].

The performance of the air-core transformer is better than the magnetic core at high frequency due to high losses in magnetic core material and low permeability. Several coreless printed circuit board (PCB) transformers have been presented in [8], with $67 \%$ transformer efficiency. However, PCB transformers are not compatible with Si-based IC technology. In [7], a $2 \mathrm{~mm}^{2}$ on-chip coreless transformer has been presented with the transformer efficiency of $70 \%$ at $170 \mathrm{MHz}$. Similarly, another air-core transformer has been presented in [9] with the efficiency of $73 \%$ at $50 \mathrm{MHz}$.
Some models have been developed for characterizing the air-core transformer. In [10] and [11], a model for stacked transformer has been proposed. It is further transformed to the spiral transformer in [12]. The model parameters were calculated using analytical expressions based on geometric parameters. These models facilitate the compilation of an optimum design in the least time for the designer. However, proximity and skin effects are not factored in, which are more pronounced at high frequency. Therefore, a thorough finite-element-based simulation is needed to model and optimize the design.

In this paper, two spiral single-layer micro-transformers are designed, simulated in the finite-element-based software COMSOL and fabricated in the Metal-MUMPs standard process.

This micro-electro-mechanical Systems (MEMS) technology greatly reduces the eddy-current losses in silicon substrate by suspending the transformer structure with a $25 \mu \mathrm{m}$ air gap. This will significantly improve the efficiency of microtransformer. Furthermore, the transformer has a single metal layer, which greatly reduces the photolithography steps and thus reduces the fabrication complexity. The designs are characterized through on-wafer testing using vector network analyzer, and the results are compared with each other in Section III. Good agreement was found between the simulated and measured results.

\section{MiCRo-TRANSFORMER DESIGN AND FABRICATION}

\section{A. Design Considerations}

The proposed micro-transformer has a 1:1 ratio and is symmetrical, as shown in Fig. 1. The spiral inductors/transformer achieves more inductance compared with meander or Hilbert shape fractal transformer in the same bounded square area [13], [14]. Therefore, symmetric transformer designs have been modeled and characterized in this paper. In symmetric transformer, two coils are symmetrically interwounded sideby-side to build a transformer structure with primary and secondary coils. In this design, only single metal layer is used in creating transformer structure. Bonding wires are used to 


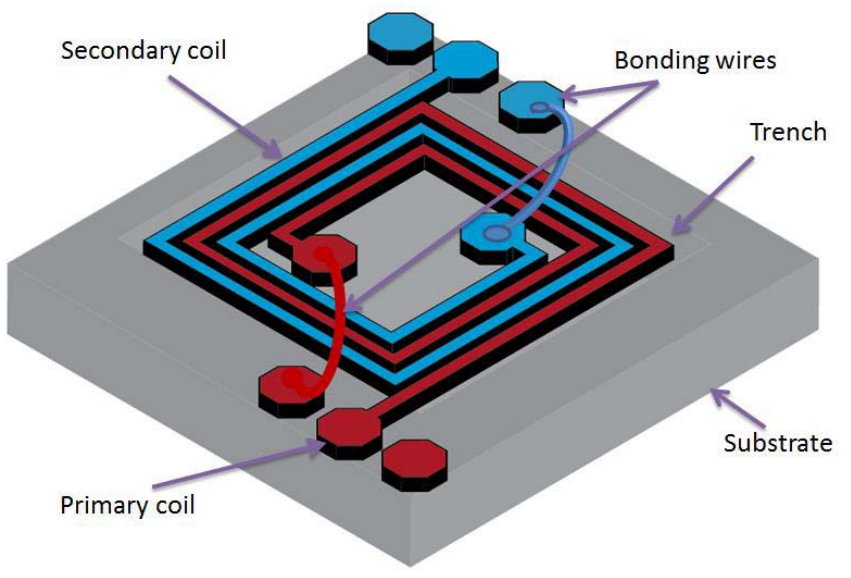

(a)

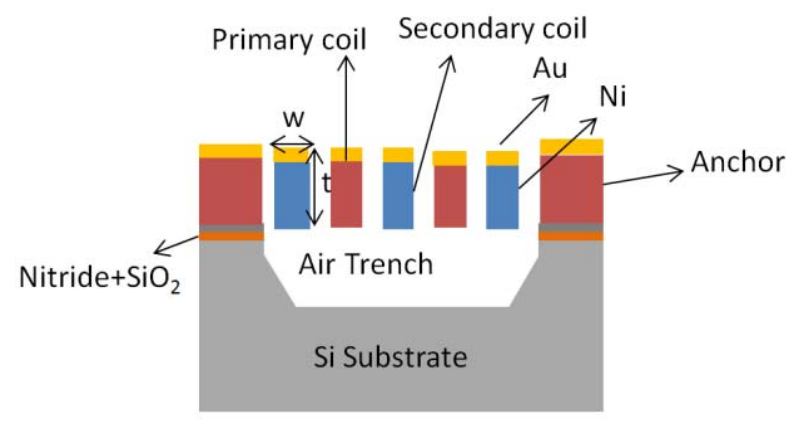

(b)

Fig. 1. Schematic layout of micro-transformer. (a) 3-D isometric view. (b) Cross-sectional view.

connect the inner terminals to the outer circuitry, as shown in Fig. 1(a).

To reduce the eddy-current loss in the substrate, a $25 \mu \mathrm{m}$ air trench is made beneath the transformer structures, as shown in Fig. 1(b). The design of a transformer in the megahertz frequency range requires high inductance with appropriate reactance. This implies a large number of transformer turns, which result in high resistance due to smaller track width and larger conductor length. To avoid high resistance coil, it is necessary to increase the thickness of the coil. Therefore, two metal layers, nickel $(20 \mu \mathrm{m})$ and gold $(0.5 \mu \mathrm{m})$, are stacked together to increase the thickness, as shown in Fig. 1(b). This technique given in [15] is used to solve the dc resistance problem which is caused by high current.

The primary/secondary inductance of symmetric transformer has been modeled in [12] as

$$
\begin{aligned}
L_{p, s} & =\frac{37.5 \times \mu_{o} \times N_{p, s}^{2} \times \mathrm{AD}^{2}}{11 \times \mathrm{OD}-14 \times \mathrm{AD}} \\
\mathrm{AD} & =\left(A_{o} \times A_{1} \times A_{2} \ldots A_{N-1}\right)^{1 / N} \\
A_{i} & =\frac{\mathrm{OD}}{2}-2 i(W+S)
\end{aligned}
$$

where $N_{p, s}$ is the number of primary and secondary turns, $\mathrm{AD}$ is the median diameter, $\mathrm{OD}$ is the outer diameter, $W$ is the width, and $S$ is the spacing between the conductors. The coupling coefficient of the symmetric spiral transformer is
TABLE I

PARAMETERS OF MICRO-TRANSFORMERS

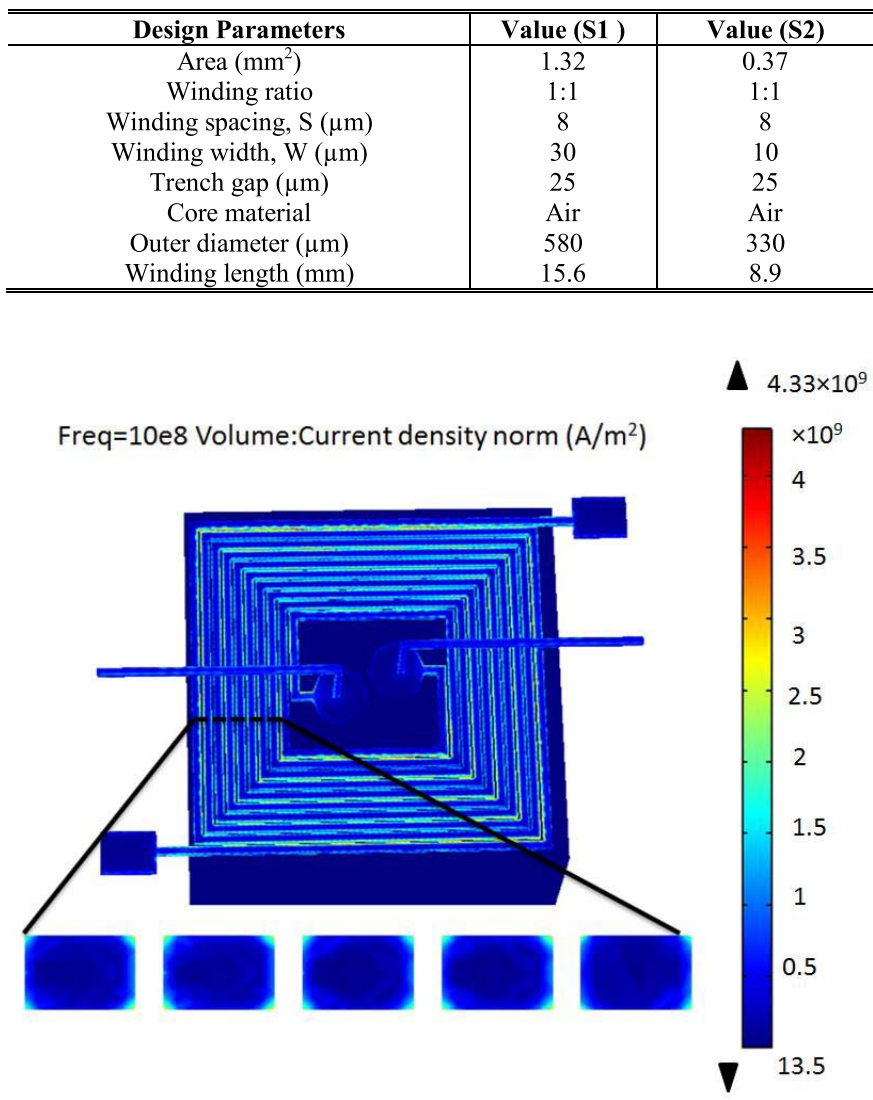

Fig. 2. Symmetric transformer current density plot. Zoomed-in view: crosssectional view of conductor.

given by

$$
k=1-\frac{(14.5 \times \mathrm{OD}-13 \times \mathrm{AD}) \times \mathrm{OD}}{37.5 \times N_{p} \times \sqrt{N_{s}} \times \mathrm{AD}^{2}} .
$$

\section{B. FEM-Based Numerical Simulation}

Two designs of symmetric micro-transformer are modeled, simulated, and fabricated with different numbers of turns, outer diameters and widths to compare the effects of these parameters on the performance of the micro-transformers. The design parameters are shown in Table I. The designs are simulated using electromagnetic module in the COMSOL multi-physics software. AC current of $1 \mathrm{~A}$ is applied to the primary coil at $1 \mathrm{GHz}$, while the secondary side is shorted for maximum load condition. The maximum current density is distributed at the outer surface and edges of the coils, as shown in Fig. 2. High magnetic field induces strong current density in the inner edges of the coils, thereby increasing the ac resistance of the primary and secondary windings. The current distribution in the coil is peaking at the edges of the conductor compared with the center, as shown in the zoomed-in view of Fig. 2. This is due to changing magnetic fields induces eddy current in the conductor, which is called the proximity effect in the transformer at high frequency.

The micro-transformer is fabricated for the first time in a low-cost Metal-MUMPs standard process. Metal-MUMPs 


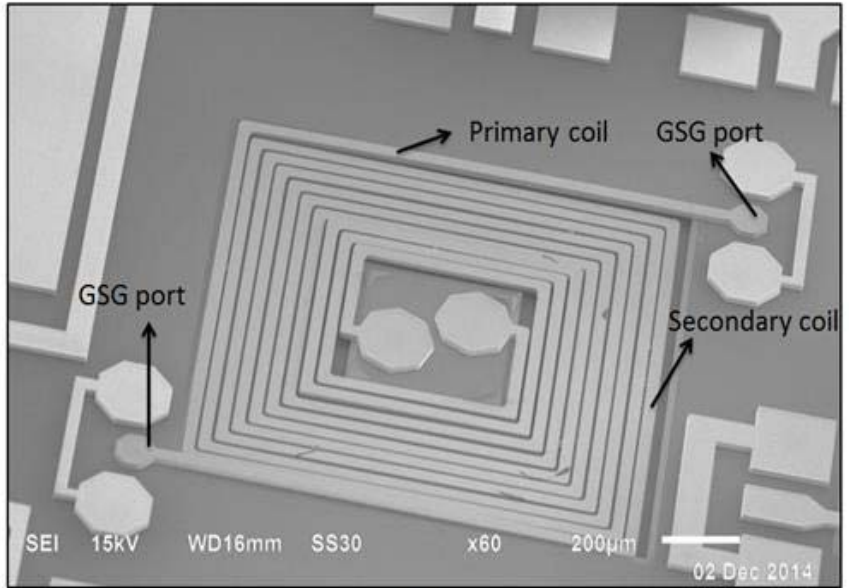

(a)

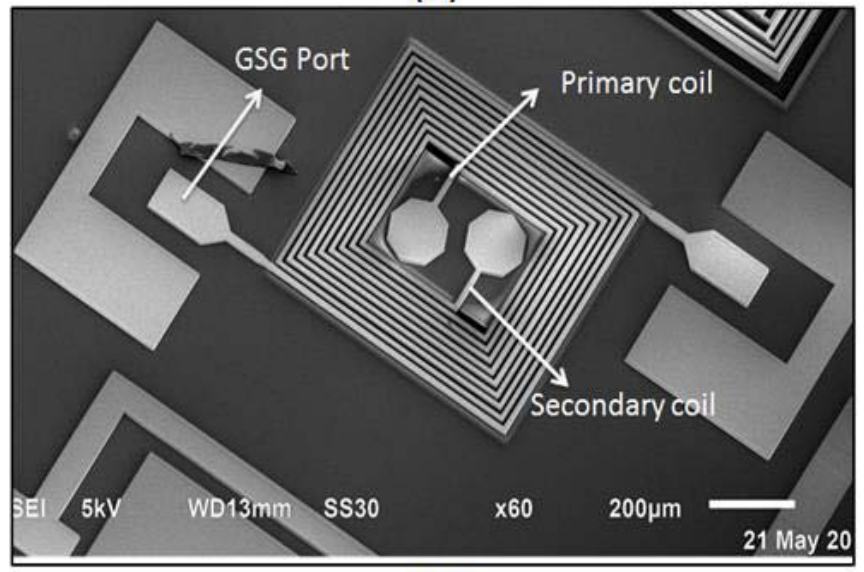

(b)

Fig. 3. SEM images of symmetric transformers. (a) $S 1$ design. (b) $S 2$ design.

is a standard process originally optimized for MEMS-based relays [16]. The SEM images of the micro-transformers are shown in Fig. 3. The design of the micro-transformer is aimed to achieve high coupling ratio, power efficiency, and quality factor. The $500 \mathrm{~nm}$ gold film on the top of $20 \mu \mathrm{m}$ nickel structure increases the conductivity and quality factor of the coils.

\section{EXPERIMENTAL RESULTS AND DISCUSSION}

\section{A. Measurement Setup}

On-wafer measurements of micro-transformers have been carried out using cascade Microtech's high-frequency probe station. The experimental setup arrangement is shown in Fig. 4. Bonding wire connections from the inside of spiral transformer to the outside terminals for measurement are shown in Fig. 4 (inset). The scattering parameters (S-parameters) were measured through the Rohde and Schwarz ZVL vector network analyzer. The errors and parasitic in cables and probes were initially removed by open, short, load and through calibration using the Agilent's on-wafer calibration tool of impedance standard substrate. The S-parameters of the devices were recorded from $20 \mathrm{MHz}$ to $1 \mathrm{GHz}$. The device pad parasitics were de-embedded from the micro-transformer using the procedure reported in [17].

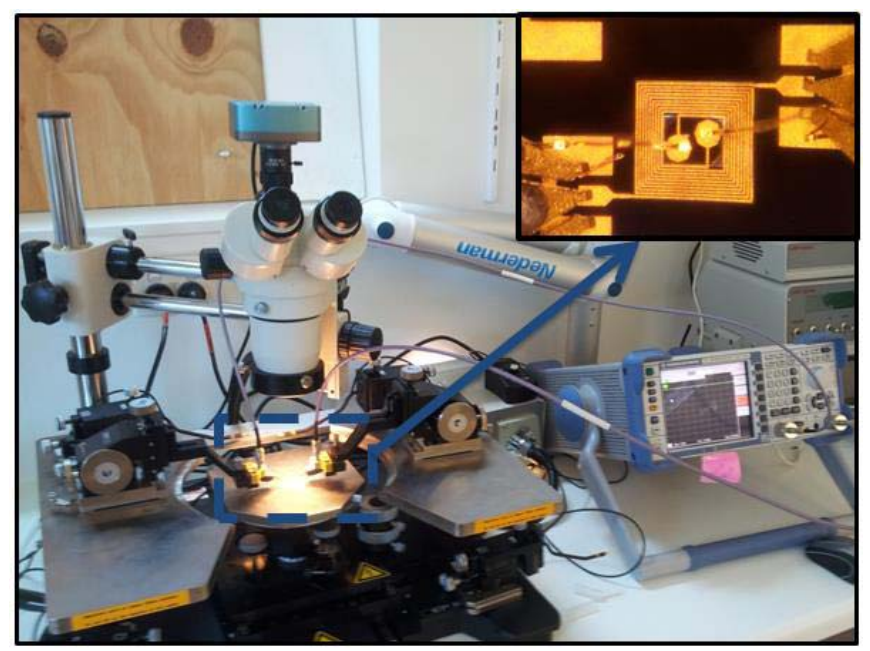

Fig. 4. Photograph of experimental setup. Inset: micro-transformer structure with bonding wires.
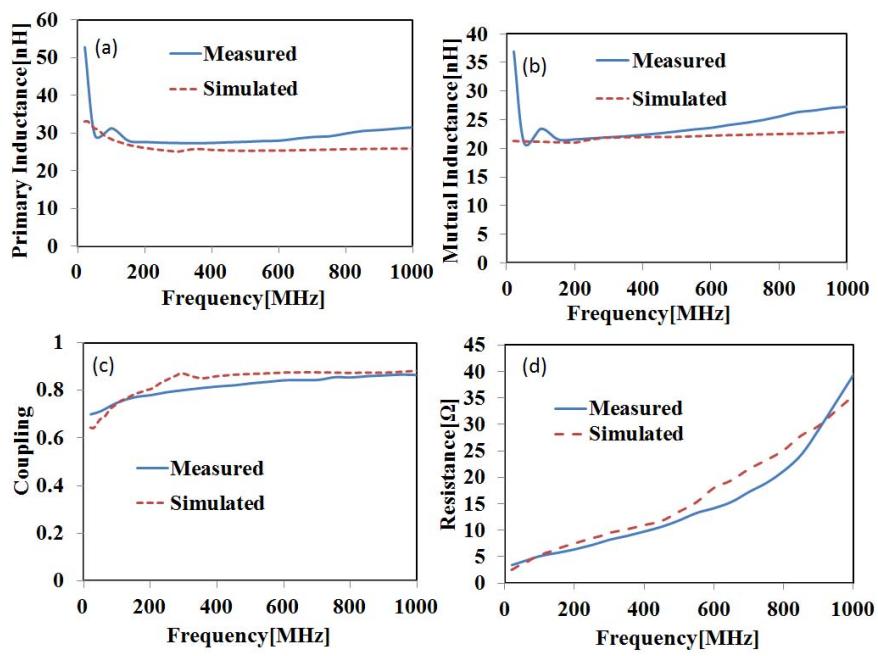

Fig. 5. Transformer characteristics versus frequency of $S 1$ design. (a) Primary inductance; (b) mutual inductance; (c) coupling; (d) resistance.

\section{B. Results and Discussions}

Fig. 3(a) shows the SEM image of $S 1$ transformer design. The dimension parameters and values are presented in Table I. The measured and simulated results of the symmetric transformer are shown in Fig. 5. The primary inductance of $51.23 \mathrm{nH}$ was measured at $20 \mathrm{MHz}$, and reduced to $31 \mathrm{nH}$ at $1 \mathrm{GHz}$. The mutual inductance of $36.39 \mathrm{nH}$ was measured at $20 \mathrm{MHz}$, and decreased to $27.12 \mathrm{nH}$ at $1 \mathrm{GHz}$. Fig. 5(c) shows the initial coupling coefficient of 0.70 at $20 \mathrm{MHz}$, which is increased to 0.87 at $1 \mathrm{GHz}$. It is observed that the coupling coefficient is almost constant above $300 \mathrm{MHz}$. The ac resistance of the transformer is shown in Fig. 5(d). The resistance rises with the increase in frequency, which is due to the skin and proximity effects.

Fig. 3(b) shows the SEM image of $S 2$ transformer design. Fig. 6 shows the characteristics of the symmetric transformer with the $10 \mu \mathrm{m}$ width of conductors. The primary inductance of $48 \mathrm{nH}$ was measured at $20 \mathrm{MHz}$, and reduced to $20 \mathrm{nH}$ at $1 \mathrm{GHz}$. The mutual inductance of 34 and $16 \mathrm{nH}$ was achieved at $20 \mathrm{MHz}$ and $1 \mathrm{GHz}$, respectively. The coupling 

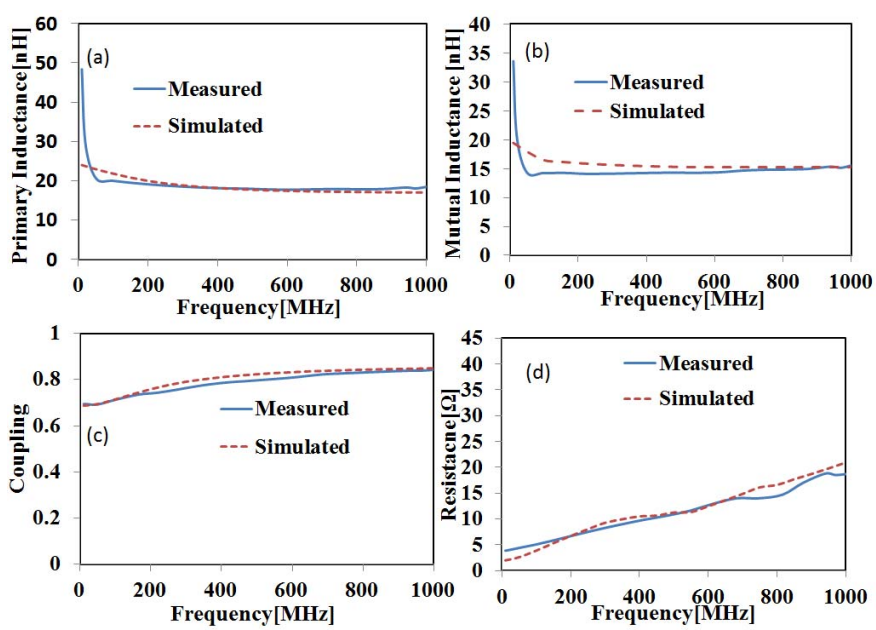

Fig. 6. Transformer characteristics versus frequency of $S 2$ design. (a) Primary inductance; (b) mutual inductance; (c) coupling; (d) resistance.

TABLE II

TRANSFORMER PARAMETERS EXTRACTED From

S-PARAMETERS MEASUREMENTS

\begin{tabular}{c|c|c}
\hline \hline Parameters & S1 design & S2 design \\
\hline Primary inductance, $L_{p} @ 1 \mathrm{GHz}[\mathrm{nH}]$ & 31.57 & 17 \\
DC resistance, $R_{d c}[\Omega]$ & 1.97 & 2.91 \\
Coupling, $k$ & 0.88 & 0.84 \\
Max. Q-factor, $Q_{\max }$ & 8.49 & 7.22 \\
Frequency of max. Q-factor, & 852 & 815 \\
$f\left(Q_{\max }\right)[\mathrm{MHz}]$ & 80.65 & 76.96 \\
Max. efficiency, $G_{\max }$ & 695 & 1000 \\
Frequency of max. efficiency, $f\left(M_{\max }\right)$ & 4.70 & 5.58 \\
Resonance frequency, $f_{\text {res }}(G H z)$ &
\end{tabular}

coefficient of the transformer is shown in Fig. 6(c), which is 0.85 at $1 \mathrm{GHz}$. The resistance of the primary coil increases linearly, and is $18 \Omega$ at $1 \mathrm{GHz}$. There is a slight difference in the simulated and experimental results, which is due to the variation in the material parameters and physical dimension of the fabricated transformer, and bonding wires influencing micro-transformer at high frequency.

The quality factor of $S 1$ design is higher than that of $S 2$ design due to larger width of $S 1$ design conductors as compared to $S 2$ design, as shown in Table II.

The power efficiency is the ratio of output load power to the power delivered to the input of the micro-transformer. The power efficiency extracted from the S-parameters for $50 \Omega$ load is given by [18]

$$
G=\frac{\left|S_{21}\right|^{2}}{1-\left|S_{11}\right|^{2}} .
$$

The maximum power efficiency is derived by assuming source, and the load is conjugately matched to the transformer, which is given as

$$
G_{\max }=\frac{\left|S_{21}\right|}{\left|S_{12}\right|}\left(K-\sqrt{\left(K^{2}-1\right)}\right.
$$

where $K$ is the Rollet stability condition defined as

$$
K=\frac{1-\left|S_{11}\right|^{2}-\left|S_{22}\right|^{2}+\left|S_{11} S_{22}-S_{12} S_{21}\right|^{2}}{2\left|S_{12} S_{21}\right|}
$$

where $S_{21}$ is the ratio of the amplitude of incident wave at the load to the incident wave on the primary coil of the

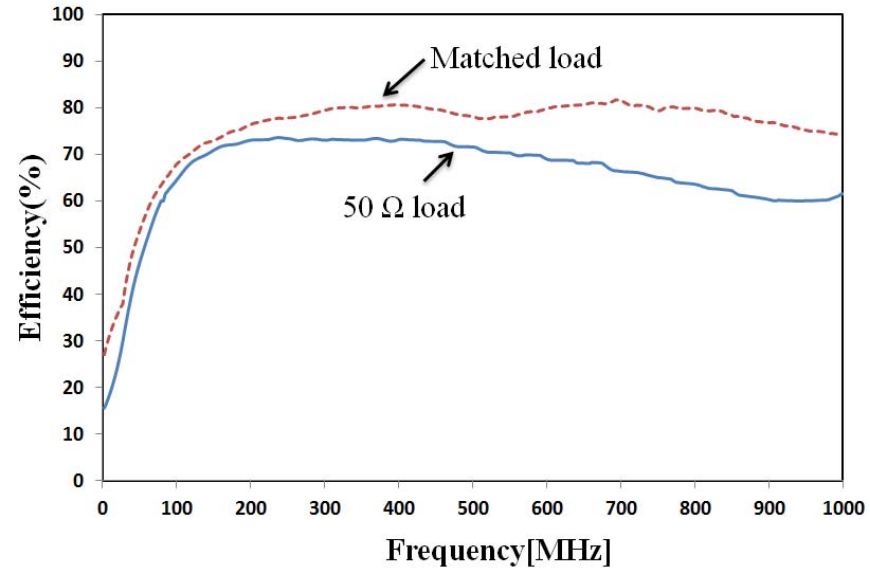

(a)

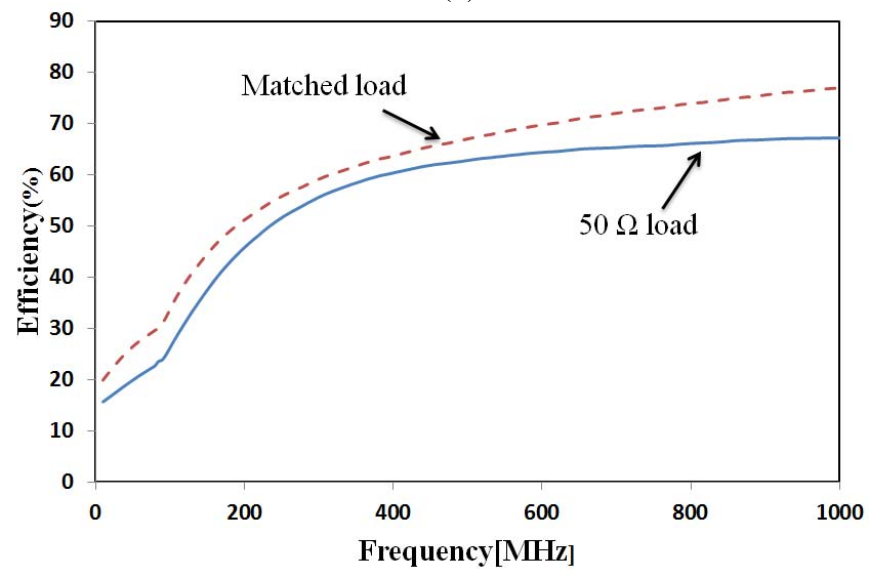

(b)

Fig. 7. Measured transformer efficiencies with $50 \Omega$ load (solid line) and matched load (dashed line). (a) $S 1$ design. (b) $S 2$ design.

micro-transformer; $S_{22}$ is the ratio of the amplitude of incident wave on the load to the reflected wave on the load of the micro-transformer. The maximum efficiency of $80.65 \%$ has been achieved at $695 \mathrm{MHz}$ assuming with the matched load for $S 1$ transformer, and $76.96 \%$ has been achieved for $S 2$ transformer at $1 \mathrm{GHz}$, as shown in Fig. 7(a) and (b). This shows that the proposed transformers have better efficiencies than the recent reported transformers [18], [19]. The reasons for the improved efficiencies are the reduced losses in silicon substrate due to air gap [18] and the reduced overall coil resistance because of the absence of vias or interconnection.

Table II shows the comparison of $S 1$ and $S 2$ transformers parameters extracted from the measured S-parameters. The primary inductance of $S 1$ transformer is almost double than that of the $S 2$ transformer due to longer length of the coil. The dc resistance of $S 2$ transformer is larger than that of $S 1$ transformer due to smaller width of the conductor. The quality factor of $S 1$ transformer is larger than that of $S 2$ due to higher inductance compared with $S 2$. Similarly, the maximum efficiency of $S 1$ transformer is higher than that of $S 2$ transformer.

Table III shows the comparison of the state-of-the-art research work in on-chip micro-transformers. It is noted that our prototypes have higher efficiencies and smallest footprints. The $S 1$ micro-transformer has better isolation properties than 
TABLE III

COMPARISON OF ON-CHIP MICRO-TRANSFORMERS

\begin{tabular}{cccccccc}
\hline \hline & Chen & Meyer & Wu & Wang & Wu & \multicolumn{2}{c}{ This work } \\
& $\mathbf{2 0 0 8}$ & $\mathbf{2 0 1 0}$ & $\mathbf{2 0 1 1}$ & $\mathbf{2 0 1 2}$ & $\mathbf{2 0 1 5}$ & S1 S2 \\
& {$[7]$} & {$[\mathbf{1 8}]$} & {$[\mathbf{9 ]}$} & {$[\mathbf{2 0}]$} & {$[\mathbf{2 1}]$} & & \\
\hline Magnetic Core & No & No & No & Yes & No & No & No \\
No. of metal layers & 2 & 2 & 1 & 1 & 1 & 1 & 1 \\
Cross-overs/Vias & Yes & Yes & Yes & No & Yes & No & No \\
Area $\left(\mathrm{mm}^{2}\right)$ & 2 & 2.3 & 2 & 3 & 2 & 1.32 & 0.37 \\
Frequency(MHz) & 170 & 125 & 50 & 20 & 20 & $20-1000$ & $20-1000$ \\
Inductance(nH) & 16 & 46 & 36 & 210 & 88 & $31 @ 1$ & $20 @ 1$ \\
& & & & & & $\mathrm{GHz}$ & $\mathrm{GHz}$ \\
Resistance $(\Omega)$ & 1.6 & 2 & 0.44 & 1.0 & 0.45 & 1.97 & 2.91 \\
Coupling & $\mathrm{N} / \mathrm{A}$ & 0.63 & 0.95 & 0.97 & 0.98 & 0.88 & 0.84 \\
Efficiency & $>70 \%$ & $78 \%$ & $73 \%$ & $75 \%$ & $80 \%$ & $80.6 \%$ & $76.6 \%$ \\
Isolation $(\mathrm{V})$ & 5000 & $\mathrm{~N} / \mathrm{A}$ & 20 & $\mathrm{~N} / \mathrm{A}$ & 380 & $>200$ & 34 \\
\hline \hline
\end{tabular}

$S 2$ micro-transformer; therefore, it is suitable for signal and power isolations.

\section{CONCLUSION}

In this paper, MEMS-based symmetric micro-transformers have been presented. The micro-transformers are optimized through the finite element analysis simulation and the implementation for the first time in the Metal-MUMPs fabrication process for isolated $\mathrm{dc} / \mathrm{dc}$ converters in the $0.02-1 \mathrm{GHz}$ frequency range. Two designs of symmetric transformer with different dimensions were characterized and compared with each other. The micro-transformers have achieved high coupling coefficients, quality factors, and efficiencies. The microtransformers utilized single metal layer, which reduced the photolithography steps and fabrication complexity. Bonding wires were used to lead out the inner terminals of microtransformer to the outer terminals. With only one layer, the transformers are suitable for single-layer power supplies.

\section{ACKNOWLEDGMENT}

This work was performed at the Queensland Node of the Australian National Fabrication Facility, a company established under the National Collaborative Research Infrastructure Strategy to provide nano- and micro-fabrication facilities for Australian researchers.

\section{REFERENCES}

[1] H. Kurata, K. Shirakawa, O. Nakazima, and K. Murakami, "Study of thin film micro transformer with high operating frequency and coupling coefficient," IEEE Trans. Magn., vol. 29, no. 6, pp. 3204-3206, Nov. 1993.

[2] M. Mino, T. Yachi, A. Tago, K. Yanagisawa, and K. Sakakibara, "Planar microtransformer with monolithically-integrated rectifier diodes for micro-switching converters," IEEE Trans. Magn., vol. 32, no. 2, pp. 291-296, Mar. 1996.
[3] M. Xu, T. M. Liakopoulos, C. H. Ahn, S. HeeHan, and H. J. Kim, "A microfabricated transformer for high-frequency power or signal conversion," IEEE Trans. Magn., vol. 34, no. 4, pp. 1369-1371, Jul. 1998.

[4] K. Yamaguchi, S. Ohnuma, T. Imagawa, J. Toriu, H. Matsuki, and K. Murakami, "Characteristics of a thin film microtransformer with circular spiral coils," IEEE Trans. Magn., vol. 29, no. 5, pp. 2232-2237, Sep. 1993

[5] A. Lotfi, R. B. Van Dover, L. Schneemeyer, and M. Steigerwald, "Micro-transformer devices using thin-film electroplated deposition," in Proc. Rec. 29th Annu. IEEE Power Electron. Specialists Conf. (PESC), May 1998, pp. 1511-1515.

[6] C. R. Sullivan and S. R. Sanders, "Design of microfabricated transformers and inductors for high-frequency power conversion," IEEE Trans. Power Electron., vol. 11, no. 2, pp. 228-238, Mar. 1996.

[7] B. Chen, "Fully integrated isolated DC-DC converter using microtransformers," in Proc. 23rd Annu. IEEE Appl. Power Electron. Conf. Expo. (APEC), Feb. 2008, pp. 335-338.

[8] S. C. Tang, S. Y. R. Hui, and H. S.-H. Chung, "Coreless planar printed-circuit-board (PCB) transformers-a fundamental concept for signal and energy transfer," IEEE Trans. Power Electron., vol. 15, no. 5, pp. 931-941, Sep. 2000.

[9] R. Wu, J. K. O. Sin, and S. Y. Hui, "Novel silicon-embedded coreless transformer for on-chip isolated signal transfer," IEEE Magn. Lett., vol. 2, Apr. 2011, Art. no. 6500103.

[10] S. S. Mohan, C. P. Yue, M. del Mar Hershenson, S. S. Wong, and T. H. Lee, "Modeling and characterization of on-chip transformers," in IEDM Tech. Dig., Dec. 1998, pp. 531-534.

[11] T. Biondi, A. Scuderi, E. Ragonese, and G. Palmisano, "Analysis and modeling of layout scaling in silicon integrated stacked transformers," IEEE Trans. Microw. Theory Techn., vol. 54, no. 5, pp. 2203-2210, May 2006.

[12] O. El-Gharniti, E. Kerherve, and J.-B. Begueret, "Modeling and characterization of on-chip transformers for silicon RFIC," IEEE Trans. Microw. Theory Techn., vol. 55, no. 4, pp. 607-615, Apr. 2007.

[13] N. Lazarus, C. D. Meyer, and S. S. Bedair, "Fractal inductors," IEEE Trans. Magn., vol. 50, no. 4, Apr. 2014, Art. no. 8400708.

[14] F. Khan, Y. Zhu, J. Lu, J. Pal, and D. V. Dao, "Micromachined coreless single-layer transformer without crossovers," IEEE Magn. Lett., vol. 6, Sep. 2015, Art. no. 6500404

[15] M. Soyuer, J. N. Burghartz, K. A. Jenkins, S. Ponnapalli, J. F. Ewen, and W. E. Pence, "Multilevel monolithic inductors in silicon technology," Electron. Lett., vol. 31, no. 5, pp. 359-360, Mar. 1995.

[16] A. Cowen, B. Dudley, E. Hill, and M. Walters, MetalMUMPs Design Handbook. Durham, NC, USA: MEMSCAP Inc., 2002.

[17] M. C. A. M. Koolen, J. A. M. Geelen, and M. P. J. G. Versleijen, "An improved de-embedding technique for on-wafer high-frequency characterization," in Proc. Bipolar Circuits Technol. Meeting, Sep. 1991, pp. 188-191.

[18] C. D. Meyer, S. S. Bedair, B. C. Morgan, and D. P. Arnold, "Highinductance-density, air-core, power inductors, and transformers designed for operation at 100-500 MHz," IEEE Trans. Magn., vol. 46, no. 6, pp. 2236-2239, Jun. 2010.

[19] A. Moazenzadeh et al., "Wire bonded 3D coils render air core microtransformers competitive," J. Micromech. Microeng., vol. 23, no. 11, p. 114020, 2013.

[20] N. Wang et al., "High efficiency Si integrated micro-transformers using stacked copper windings for power conversion applications," in Proc. 27th Annu. IEEE Appl. Power Electron. Conf. Expo. (APEC), Feb. 2012, pp. 411-416.

[21] R. Wu, N. Liao, X. Fang, and J. K. O. Sin, "A silicon-embedded transformer for high-efficiency, high-isolation, and low-frequency on-chip power transfer," vol. 62, no. 1, pp. 220-223, Jan. 2015. 